\title{
Produção do "leite" de junça (cyperus esculentus) via planejamento de experimentos
}

\author{
${ }^{1}$ J. J. G. da COSTA NETO, P. F. F. AMARAL, G. C. FONTES, M. H. M. ROCHA \\ LEÃO. \\ ${ }^{1}$ Universidade Federal do Rio de Janeiro. Departamento de Engenharia Bioquímica \\ E-mail para contato: jonasneto@ifma.edu.br
}

RESUMO: A junça, tiger nuts ou chufa é um tubérculo pertencente a família das Cyperáceas. Desse tubérculo se extrai um extrato chamado de "leite" de junça no Brasil ou "Horchata de chufa" na Espanha, bebida não alcoólica com grande comercialização, principalmente, na região de Valência (40 a 50 milhões de litros), que ainda carece de uma produção em escala industrial que considere, principalmente, melhores condições nutricionais. Portanto, o objetivo foi avaliar a extração do leite de junça por meio de uma investigação exploratória quanto às condições experimentais estatisticamente significativas para as variáveis respostas rendimento e teor de proteína. Para avaliação das condições experimentais, foi realizado um planejamento fracionário para definir as variáveis independentes significantes (tempo de saturação, temperatura de saturação, concentração de metabisulfito de sódio e tempo de moagem). Os resultados indicaram que apenas os fatores concentração de metabisulfito e sua interação com o tempo de saturação foram marginalmente significativos para a variável resposta rendimento. Para a proteína não houve fatores e interações significativos $(p<0,05)$

\section{Introdução}

Segundo Marx (1985) e Okoli (1996), a junça, juncinha, tiririca-amarela, tiger nuts ou chufa, Cyperus esculentus, da família das Cyperáceas, é cultivada principalmente em solos arenoso. A planta é originária da parte leste da África e sua utilização é muito antiga. A junça produz tubérculos com um núcleo amarelado cercado por uma envoltura fibrosa de coloração marrom. Os tubérculos são doces ao paladar e são ricos em carboidratos e gorduras, são comestíveis e não se possui casos notificados sobre toxicidade

Selma (2003), Morell (1983) CRDO (2012) e Navarro (1984) mostraram que o "leite de junça" ou "horchata" um subproduto extraído da junça, é uma bebida não alcóolica muito consumida na Espanha e em alguns países africanos, cuja utilização vem se expandindo bastante pelo mundo. Na Espanha, movimenta 60 milhões de euros, o que corresponde aproximadamente a 40-50 milhões de litros por ano. Possui baixa acidez (6,3-6,8) e alta qualidade nutricional, o que aumenta seu potencial quanto à comercialização. É um produto rico em amido, ácido oleico, linoleico, proteínas e aminoácidos, principalmente, arginina. 
Neste trabalho foi realizado um planejamento experimental fracionário $\left(2^{\mathrm{k}-\mathrm{p}}\right)$ para investigação exploratória da significância dos fatores em estudo quanto ao teor de proteína e rendimento para produção do "leite" de junça

\section{Materiais e métodos}

\subsection{Obtenção da junça}

O experimento envolveu uma amostragem completamente aleatória de uma variedade de junça (cyperus esculentos): com diâmetro de 0,5-1,0cm, previamente seca, obtida no município de Morros-MA, a leste da capital maranhense. O tubérculo foi lavado e estocado sob refrigeração $\left(4^{\circ} \mathrm{C}\right)$.

\subsection{Produção do leite de junca}

Para produção do leite foram utilizados $20 \mathrm{~g}$ de junça previamente sanitizadas e saturadas sob uma solução de metabisulfito de sódio $\left(\mathrm{Na}_{2} \mathrm{~S}_{2} \mathrm{O}_{5}\right)$ Após a saturação a junca foi lavada com água destilada e em seguida moída em um liquitificador industrial (Fak metalurgica $800 \mathrm{~W}$ ). Posteriormente, para remoção do material sólido e obtenção do leite, utilizou-se uma peneira de aço inox ASTM 1/4 polegadas com abertura de $0,2 \mathrm{~mm}$. O procedimento de extração foi realizado como mostra a Figura 1.

Para otimização da produção do leite foi aplicado o método do planejamento experimental fracionário $2^{4-1}$, com o uso do programa de computação Statistica 7.0. Os parâmetros fixados e adotados como variáveis independentes foram: concentração de metabisulfito de sódio, tempo de saturação, tempo de moagem e temperatura de saturação. A Tabela 1 apresenta os valores que representam os limites para cada parâmetro estudado. Vale ressaltar que o intervalo de temperatura variou de $25-55^{\circ} \mathrm{C}$, sendo o último definido previamente até $55^{\circ} \mathrm{C}$ pelo fato de que temperaturas superiores causam a gelatinização do amido com consequente perda sensorial do "leite'(CORTÉS, 2004; DJOMDI, 2005; EJOH, 2005). A Tabela 2 mostra os experimentos a serem realizados no planejamento experimental.

As variáveis de respostas em estudo foram: rendimento $(\%)$ e proteína $(\mathrm{mg} / \mathrm{ml})$, $\mathrm{O}$ rendimento foi calculado por diferença de peso da matéria seca da junça. A proteína foi analisada segundo o Método de Bradford (1976).

A análise estatítica foi realizada pelo softwere Statistica 7.0

Tabela 1 - Fatores e níveis estudados para extração do "leite" de junça

\begin{tabular}{ccccc}
\hline & Fatores & Níveis & +1 \\
\hline $\mathrm{MNa}$ & {$\left[\mathrm{Na}_{2} \mathrm{~S}_{2} \mathrm{O}_{5}\right](\%)$} & 0,025 & 0,075 & 0,125 \\
Te.S & Tempo de saturação $(\mathrm{h})$ & 8 & 14 & 20 \\
T.S & Temperatura de saturação $\left({ }^{\circ} \mathrm{C}\right)$ & 25 & 40 & 55 \\
T.M & Tempo de moagem $($ min $)$ & 5 & 17,5 & 30 \\
\hline
\end{tabular}


Tabela 2 - Condição de operação do experimento com três pontos centrais

\begin{tabular}{ccccc}
\hline Ensaio & $\begin{array}{c}{\left[\mathrm{Na}_{2} \mathrm{~S}_{2} \mathrm{O}_{5}\right]} \\
(\%)\end{array}$ & $\begin{array}{c}\text { Tempo de } \\
\text { saturação } \\
(\mathrm{h})\end{array}$ & $\begin{array}{c}\text { Temperatura } \\
\text { de } \\
\text { saturação } \\
\left({ }^{\circ} \mathrm{C}\right)\end{array}$ & $\begin{array}{c}\text { Tempo } \\
\text { de } \\
\text { moagem } \\
(\mathrm{min})\end{array}$ \\
\hline 1 & 0,025 & 8,000 & 25,000 & 5,000 \\
2 & 0,125 & 8,000 & 25,000 & 30,000 \\
3 & 0,025 & 20,000 & 25,000 & 30,000 \\
4 & 0,125 & 20,000 & 25,000 & 5,000 \\
5 & 0,025 & 8,000 & 55,000 & 30,000 \\
6 & 0,125 & 8,000 & 55,000 & 5,000 \\
7 & 0,025 & 20,000 & 55,000 & 5,000 \\
8 & 0,125 & 20,000 & 55,000 & 30,000 \\
9 & 0,075 & 14,000 & 55,000 & 17,500 \\
10 & 0,075 & 14,000 & 55,000 & 17,500 \\
11 & 0,075 & 14,000 & 55,000 & 17,500 \\
\hline
\end{tabular}

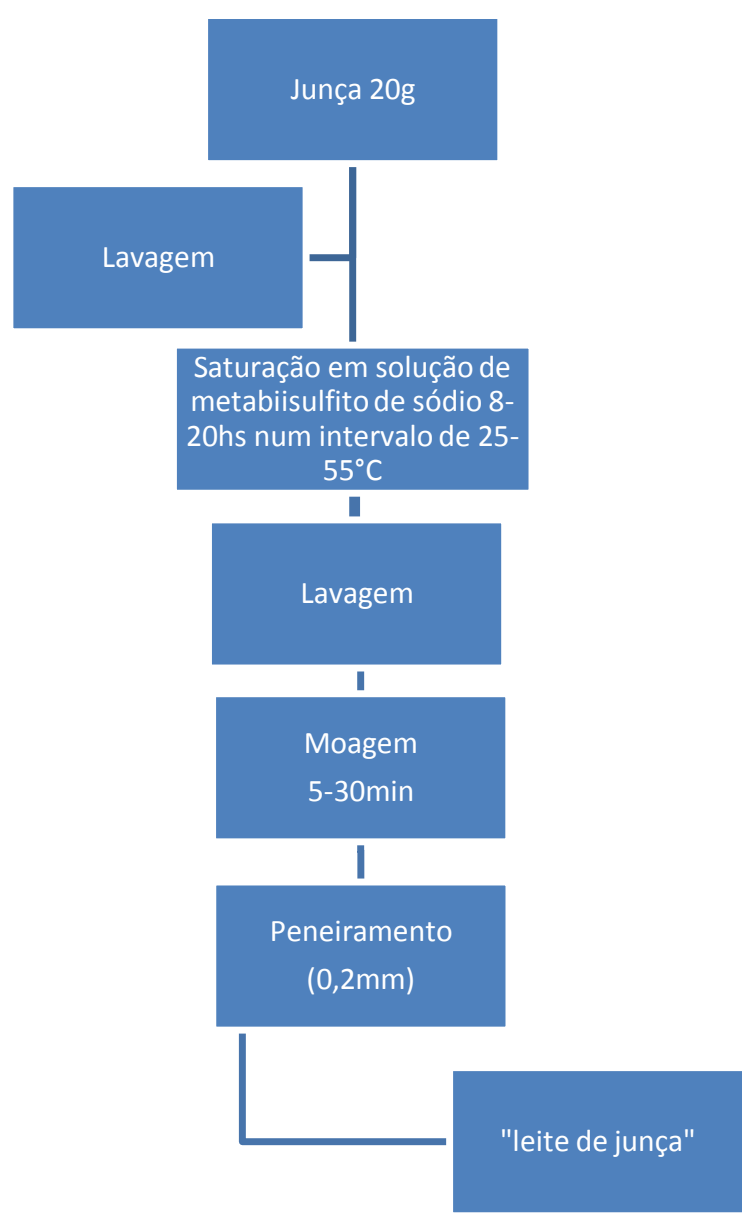

Figura 1 - Produção do leite de junça 


\section{RESULTADO E DISCUSSÃO}

Inicialmente foi possível avaliar que os dos dados obtidos, conforme as condições do processo, para o rendimento variaram de 22,40-29,60 \% e para proteína de $0,773-3,914 \mathrm{mg} / \mathrm{L}$ como mostra a Tabela 3.

Tabela 3 Matriz de planejamento $2^{4-1}$ com 3 repetições no ponto central e as variáveis respostas estudadas: Rendimento e proteína

\begin{tabular}{cccccc}
\hline & \multicolumn{2}{c}{ Fatores } & & \multicolumn{2}{c}{ Respostas } \\
{$[\mathrm{MNa}](\%)$} & Te.S $(\mathrm{h})$ & T.S $\left({ }^{\circ} \mathrm{C}\right)$ & T.M $(\mathrm{min})$ & Rendimento $(\%)$ & Proteína (mg/L) \\
\hline 0,025 & 8,00 & 25,00 & 5,00 & 23,36 & 1,862 \\
0,125 & 8,00 & 25,00 & 30,00 & 29,60 & 2,701 \\
0,025 & 20,00 & 25,00 & 30,00 & 24,50 & 3,583 \\
0,125 & 20,00 & 25,00 & 5,00 & 22,50 & 0,773 \\
0,025 & 8,00 & 55,00 & 30,00 & 23,91 & 1,644 \\
0,125 & 8,00 & 55,00 & 5,00 & 26,67 & 1,974 \\
0,025 & 20,00 & 55,00 & 5,00 & 23,83 & 1,855 \\
0,125 & 20,00 & 55,00 & 30,00 & 22,49 & 2,491 \\
0,075 & 14,00 & 40,00 & 17,50 & 24,98 & 1,241 \\
0,075 & 14,00 & 40,00 & 17,50 & 23,07 & 1,212 \\
0,075 & 14,00 & 40,00 & 17,50 & 25,23 & 3,914 \\
\hline
\end{tabular}

$\mathrm{MNa}=$ Concentração de metabisulfito; Te.S = Tempo de saturação; TS = Temperatura de saturação; $\mathrm{TM}=$ Tempo de moagem.

Para avaliar os fatores que exerceram maior influência nas variáveis respostas, rendimento e proteína, realizou-se a análise de variância (ANOVA) como mostra as Tabelas 4 e 5 respectivamente

Tabela 4 Análise de variância (ANOVA) do modelo ajustado para o rendimento $\left(\mathrm{R}^{2}=0,93\right)$

\begin{tabular}{cccccc}
\hline Fator & SS & g.L & M.S & F & P \\
\hline 1 MNa & 4,00445 & 1 & 4,00445 & 2,86845 & 0,232412 \\
2 Te.S & 13,05605 & 1 & 13,05605 & 9,35225 & 0,092353 \\
3 T.S & 1,17045 & 1 & 1,17045 & 0,83841 & 0,456511 \\
4 T.M & 2,14245 & 1 & 2,14245 & 1,53467 & 0,341079 \\
$1-2$ & 19,03445 & 1 & 19,03445 & 13,63467 & 0,066148 \\
$1-3$ & 0,99405 & 1 & 0,99405 & 0,71205 & 0,487602 \\
$1-4$ & 0,36125 & 1 & 0,36125 & 0,25877 & 0,661530 \\
Erro puro & 2,79207 & 2 & 1,39603 & & \\
Total SS & 43,62656 & 10 & & & \\
\hline
\end{tabular}


Tabela 5 - Análise de variância (ANOVA) do modelo ajustado para a proteína $\left(\mathrm{R}^{2}=0,50\right)$

\begin{tabular}{cccrccc}
\hline Fator & SS & g.L & M.S & F & $p$ \\
\hline $1 \mathrm{MNa}$ & 0,000295 & 1 & & 0,000295 & 0,000123 & 0,992168 \\
$2 \mathrm{Te} . \mathrm{S}$ & 0,124750 & 1 & & 0,124750 & 0,051908 & 0,840949 \\
3 T.S & 0,032896 & 1 & & 0,032896 & 0,013688 & 0,917553 \\
$4 \mathrm{~T} . \mathrm{M}$ & 0,116886 & 1 & 0,116886 & 0,048636 & 0,845920 \\
$1-2$ & 1,961190 & 1 & 1,961190 & 0,816040 & 0,461685 \\
$1-3$ & 1,395285 & 1 & 1,395285 & 0,580571 & 0,525682 \\
$1-4$ & 1,079715 & 1 & 1,079715 & 0,449264 & 0,571715 \\
Erro puro & 4,806600 & 2 & 2,403300 & & \\
Total SS & 9,629719 & 10 & & & \\
\hline
\end{tabular}

Com base na Tabela 4 notou-se que todos os fatores na faixa estudada não foram estatisticamente significativos. Porém, os fatores tempo de saturação e a interação entre o tempo de saturação e concentração de metabisulfito de sódio foram marginalmente significativos $(0,05 \leq \mathrm{p} \leq 0,1)$ em relação ao rendimento para um coeficiente de determinação que explica $93,6 \%$ da variação em torno da média. Contudo, o seguinte modelo não apresentou normalidade satisfatória como pode ser observado na Figura 3, uma vez que os resíduos não seguem uma distribuição normal, conforme o teste de Shapiro wilk ( $p>0,05)$ cujo valor foi $\mathrm{p}=0,0004$

Para a variável resposta proteína, nenhum dos fatores e interações em estudo, como mostra a tabela 5 , foram significativos $(\mathrm{p}<0,05)$ para faixa estudada, além de possuir uma baixa explicação $(50 \%)$ da variação em torno da média e não apresentar uma normalidade satisfatória $\mathrm{p}=0,00024$ (Figura 4), conforme o teste de Shapiro Wilk $(\mathrm{p}>0,05)$.

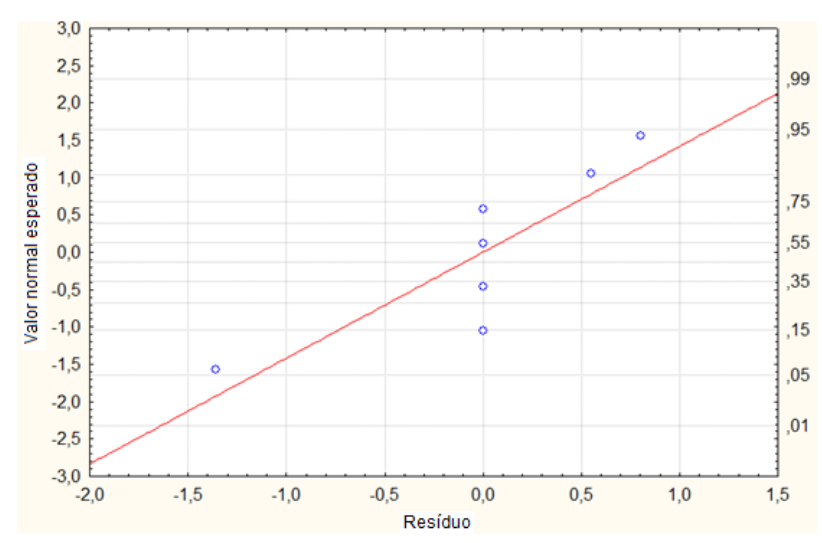

Figura 3-Probabilidade normal dos resíduos para o rendimento do processo de obtenção do "leite" de junça

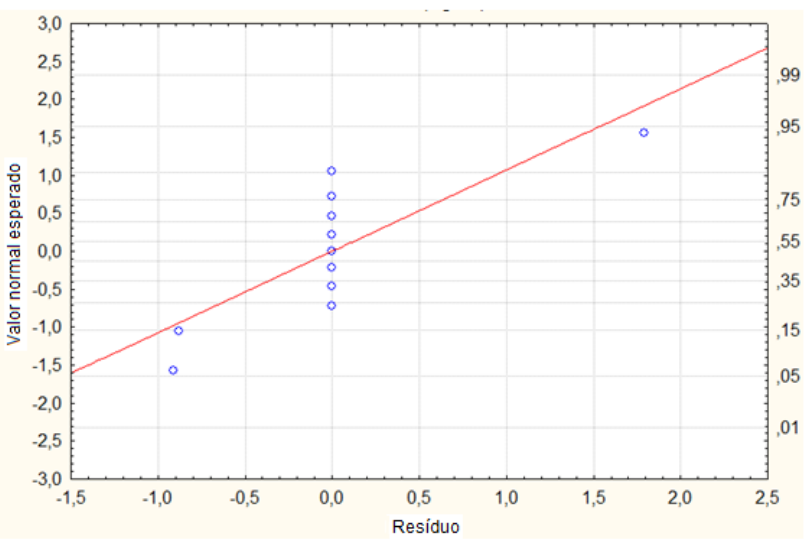

Figura 4-Probabilidade normal dos resíduos para o teor de proteína do processo de obtenção do "leite" de junça 
Avaliando os resultados obtidos, notou-se que nos intervalos estudados, os maiores rendimentos na obtenção do "leite" ocorreram para maiores níveis de metabisulfito de sódio $(0,125 \%)$ e tempo de moagem $(30 \mathrm{~min})$ e, nos menores níveis de temperatura $\left(25^{\circ} \mathrm{C}\right)$, temperatura $\left(25^{\circ} \mathrm{C}\right)$ e tempo $(8 \mathrm{hs})$ de saturação, com rendimento máximo de $29,60 \%$. Fato que ocorreu de maneira oposta ao observado por Ejoh, (2005), quanto aos seus fatores de estudo, ou seja, tempo de saturação e temperatura de saturação, cujos ensaios foram realizados de forma aleatoria. A temperatura e o tempo máximo de saturação utilizada pelo autor foi de $100^{\circ} \mathrm{C}$ e $50 \mathrm{hs}$ respectivamente, onde o melhor rendimento encontrado $(19,61 \%)$ aconteceu nos maiores níveis de tempo e temperatura de saturação, desconsiderando as características sensoriais do "leite" em virtude da gelatinização do amido. Além disso, estes resultados foram opostos, quando comparados ao comportamento de extração do "leite" de soja de acordo com Johson e Snyder (1978), que obtiveram resultados semelhantes ao de Ejoh (2005).

Para obtenção do ponto ótimo de rendimento é necessário realizar um estudo conjunto através do planejamento de composto central, levando em consideração os fatores marginalmente significativos estatisticamente conforme observado na Tabela 4. Pode-se ascender a faixa de trabalho para o tempo de saturação, porém não é possível o mesmo com o fator temperatura de saturação, pois têm-se um limite de $55^{\circ} \mathrm{C}$ para que o "leite" não sofra perda sensorial com a consequente gelatinização do amido.

Para a variável proteína, não foi possível obter resultados conclusivos, uma vez que não houve fatores e interações significativos $(\mathrm{p}<0,05)$

\section{Considerações finais}

Por meio da análise exploratória via planejamento experimental fracionário, foi possível definir os fatores marginalmente significativos como concentração de metabisulfito de sódio e tempo de saturação para a variável resposta rendimento, bem como a influência dos outros fatores nessa variável. Apesar disso, não foi possível definir os pontos ótimos, para obtenção do "leite" sendo necessário um estudo conjunto utilizando o planejamento de composto central, com base nos fatores significativos para esta variável. Quanto à variável proteína, percebeu-se que os fatores em estudos não influenciaram significativamente este nutriente dentro da faixa estudada, visto que não houveram fatores estatisticamente significativos, além de um modelo de baixa explicação $(50 \%)$ e que não configura uma distribuição normal. Assim, utilizando futuros planejamentos de composto central rotacional, espera-se encontrar as melhores condições para extração do "leite" de junça, baseando-se em um processo padronizado com melhor rendimento, para que a mesma possa ser comercializada mais facilmente em escala industrial e assim atender demandas futuras no Brasil.

\section{Referências Bibliográficas}

ASSOCIATION OF OFFICIAL ANALYTICAL CHEMISTS INTERNATIONAL (AOAC). Official methods of analysis Chemists. 16. ed. Gaitherburg: AOAC, v.2, cap. 32, p.1-43. 1997.

BRADFORD, M. Analytical Biochemistry. 72, 248-254, 1976.

CRDO: Consejo Regulador de la Denominaci'on de Origen Chufa de Valencia. 2013. Acessível em: http://www.chufadevalencia.org. Acessado em 06 de agosto de 2013. 
EJOH, R. A, DJONDI, NDJOUENKEU, R., Characteristics of tigernuts (cyperus esculentus) tubers and their performance in the production of a milk drink. Journal od Food Processing and preservation. n.30, p.145-163, 2006

JOHNSON, K. W, SNYDER, H. E, Soymilk: A comparison of processing methods on yields and composition. J. Food Sci., n.43, p. 349-353, 1978.

MARX, F., KERR, W. E. Junça (Cyperus esculentus Linné), bulbilho consumido no maranhão e que possui bom valor nutricional. Acta Amazônica, n.15 p.1-2, 1985.

MORELL J. \& BARBER S. "Chufa y Horchata: Características Físicas, Químicas y Nutritivas". Caja de Ahorros de Valencia, Departamento de investigación y desarrollo agroindustrial, IATA (CSIC). 1983.

NAVARRO J.L. SCHWARTZ M., GASQUE F., ALBEROLA J., PÉREZ R. and LAFUENTE B. Influencia de la época de recolección de la chufa (Cyperus esculentusL.) sobre las características analíticas y sensoriales de la horchata. Rev. Agroquim. Tecnol. Aliment. n.24 p.199, 1984.

OKOLI, C. A. N., SHILling, D. G., SMITH, R. L., BEWWICK, T. A. Genetic diversity in purple nutsedge (Cyperus esculentus L.). Biological control, 1997. 